\title{
Nachruf auf Karl-Heinz Schulmeyer
}

Am 11.10.2013 ist Karl-Heinz Schulmeyer nach kurzer schwerer Krankheit verstorben. Wir trauern sehr um unseren hochgeschätzten Kollegen, der auch über die Grenzen des Palmengartens hinaus vielen Kolleginnen und Kollegen in anderen botanischen Gärten als äußerst kompetenter und erfahrener Bromeliengärtner bekannt ist.

Karl-Heinz Schulmeyer wurde am 15.11.1955 in Mörfelden-Walldorf geboren, wo er auch zuletzt noch wohnte. Seine Lehre als Chemielaborant bei den Farbwerken Hoechst hat er kurz nach Beginn abgebrochen. Daraufhin trat er zum nächstmöglichen Termin in den Dienst der Stadt Frankfurt ein und begann am 2.1.1973 in der Stadtgärtnerei Frankfurt seine Ausbildung als Gärtner. Nach erfolgreich abgeschlossener Lehre wurde er in der heute nicht mehr existierenden Stadtgärtnerei eingestellt. Der Beruf des Gärtners und die Kultur von Pflanzen begeisterten ihn, er war wissbegierig und bildete sich stets weiter. 1990 erhielt er in der Stadtgärtnerei eine Fachvorarbeiterstelle und war dort verantwortlich für die Topf- und Beetpflanzenanzucht. Schon damals war KARLHeinz Schulmeyer ein äußerst verantwortungsbewusster Mitarbeiter, dem das Wohl der Kolleginnen und Kollegen sowie die Sicherheit am Arbeitsplatz sehr wichtig waren. Im Jahr 1985 wurde er als Sicherheitsbeauftragter für die Gärtnerei bestellt. Auch in seiner Freizeit setzte er sich für das Allgemeinwohl ein und war seit seiner Jungend Mitglied der Freiwilligen Feuerwehr Mörfelden.

Im Zuge der Schließung der Stadtgärtnerei wechselte Karl-Heinz Schulmeyer am 1.7.1994 zum Palmengarten. Zum 1.2.1995 wurde ihm die Stelle eines Spezialisten für botanische Typensammlung und die Betreuung des Bromelienhauses angeboten. Seit dieser Zeit betreute er die Bromeliensammlung des Palmengartens.

Die Übernahme einer großen wissenschaftlich wertvollen Bromeliensammlung war eine

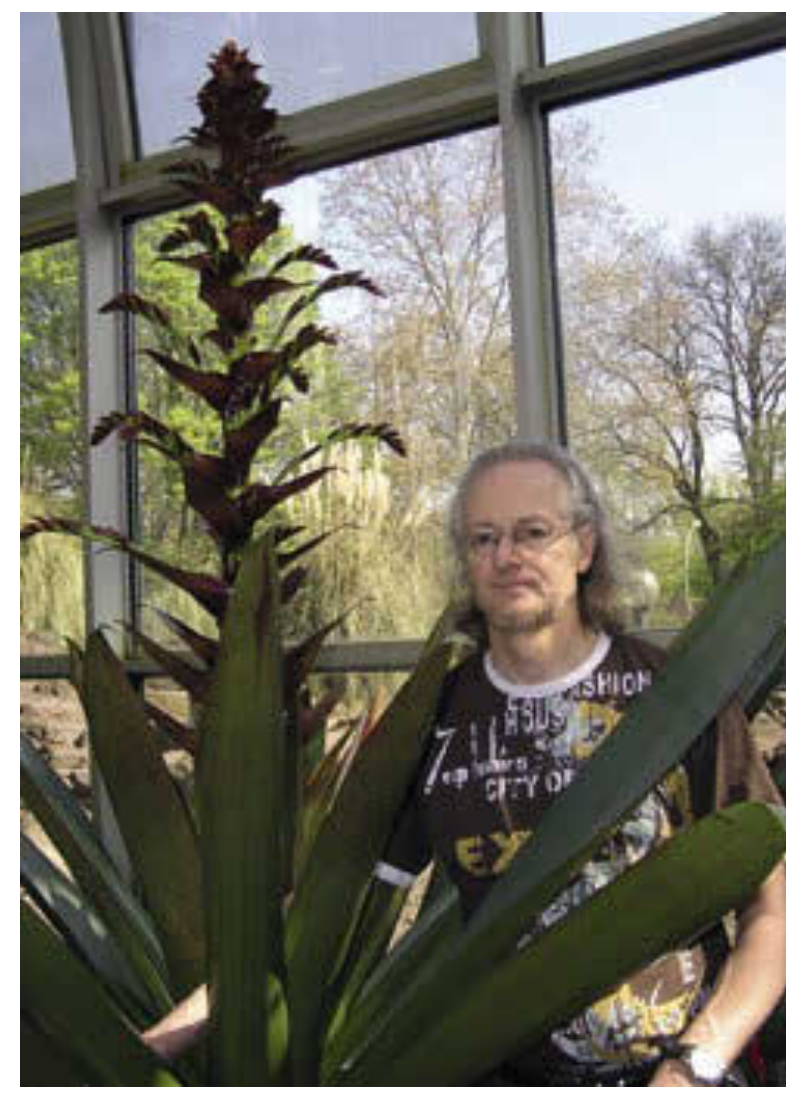

Karl-Heinz Schulmeyer in seinem Element.

große Herausforderung. Seine Begeisterung für die Bromelien war groß, schnell arbeitete sich Karl-Heinz Schulmeyer in die Materie ein und war bald ein ausgewiesener Bromelienspezialist. Er nutzte jede Gelegenheit, sein Fachwissen zu erweitern, nahm an Fortbildungen des Verbandes Botanischer Gärten teil, war Mitglied der Bromeliengesellschaft und pflegte regelmäßig Kontakte zu Fachleuten, die er im In- und Ausland besuchte. Jede Gelegenheit wurde genutzt, um im Anschluss an Tagungen noch andere botanische Gärten zu besuchen und dort Kolleginnen und Kollegen zu treffen. Zwei Mal reiste er zusammen mit Kolleginnen und Kollegen aus dem Palmengarten nach Brasilien. Für ihn war es ein besonderes Erlebnis, die Bromelien an ihrem natürlichen Standort zu erkunden. Die erworbenen Kenntnisse ließ 


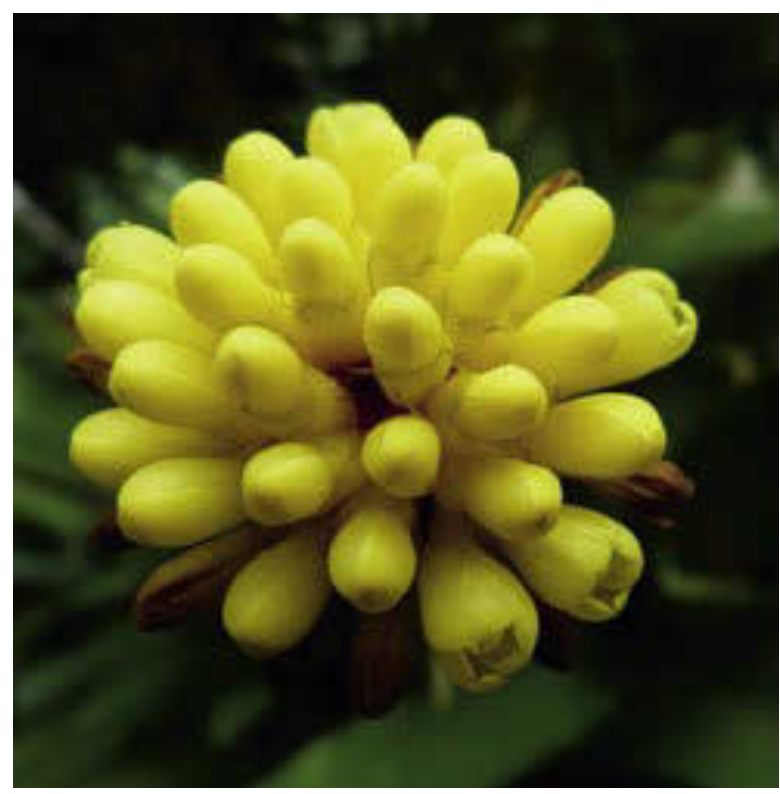

Aufsicht auf den Blütenstand von Aechmea kleinii im Bromelienhaus.

er in die Arbeit im Palmengarten einfließen. Wenn er bei den Exkursionen etwas Besonderes sehen wollte, scheute er keine Mühe und war öfter plötzlich ,irgendwo in der Botanik verschwunden".

Er brachte von diesen Reisen auch Samen besonderer Bromelien mit. Ein Teil der daraus hervorgegangenen Pflanzen existiert noch heute im Palmengarten. Seine besondere Liebe galt nicht ausschließlich den Bromelien, er begeisterte sich auch für andere Pflanzen wie z.B. Passifloren, von denen er einige Arten im Bromelienhaus kultivierte. Gerne gab er sein Wissen weiter, machte häufig öffentliche Führungen zum Thema Bromelien und lieferte fachliche Hinweise für Beiträge für die Palmengarten-Zeitschrift. Ferner engagierte er sich seit Jahren im biologischen Pflanzenschutz und hat dabei auch eine Nützlingszucht aufgebaut.

Karl-Heinz Schulmeyer dachte stets an das Wohl der Kolleginnen und Kollegen des Palmengartens und war ein besonders sozial denkender und handelnder, hilfsbereiter Mensch. Ihn störte es nicht, wenn er mit seiner Meinung manchmal gegen den Strom schwamm und dabei auch aneckte. Geradlinig und unbeirrbar verfolgte er alles, was ihm wichtig war, auch wenn es Gegenwind gab. Ein besonderes Anliegen waren ihm auch Belange der Arbeitssicherheit und des Brandschutzes. Dank seiner Tätigkeit bei der Freiwilligen Feuerwehr war er uns auf diesem Gebiet stets ein sehr kompetenter und kritischer Berater. Zuletzt hatte er sich insbesondere für das altersgerechte Arbeiten eingesetzt und sich in einer dazu neu gegründeten Arbeitsgruppe engagiert.

Über viele Jahre hinweg leistete er als Mitglied des Personalrates und der Gewerkschaft wertvolle Arbeit. Auch hierzu besuchte er zahlreiche Fortbildungsveranstaltungen. Sein Wissen ist uns allen zugutegekommen. Wann immer Hilfe benötigt wurde, war er zur Stelle, auch für die Organisation von Betriebsausflügen oder Weihnachtsfeiern setzte er sich ein. Karl-Heinz Schulmeyer war belesen und interessierte sich unter anderem für Stadtgeschichte.

Karl-Heinz Schulmeyer feierte gern zusammen mit Kolleginnen und Kollegen. $\mathrm{Zu}$ Beginn des Jahres 2013 konnte er sein 40-jähriges Dienstjubiläum begehen. Am 7.2. hatte er zur feierlichen Übergabe der Jubiläumsurkunde die ganze Belegschaft eingeladen. In einer spannenden und unterhaltsamen Rede trug er seinen Werdegang vor und zeigte damit eindrücklich, wie sehr er sich mit dem Palmengarten verbunden fühlte.

Die letzten Augenblicke seines Lebens hat Karl-Heinz Schulmeyer im Palmengarten verbracht, für ihn ein besonders vertrauter und geliebter Ort. Er hinterlässt viele Spuren, nicht nur die von ihm herangezogenen und gepflegten Pflanzen. Noch lange werden sicherlich auch die von ihm mit seiner charakteristischen Handschrift versehenen Pflanzenetiketten überdauern.

Die durch seinen Tod entstandene Lücke wird schwer zu schließen sein. Wir werden ihn nicht vergessen. 